The Astrophysical Journal, 186:149-163, 1973 November 15

(C) 1973. The American Astronomical Society. All rights reserved. Printed in U.S.A.

\title{
OPTICAL INTERSTELLAR LINES IN DARK CLOUDS
}

\author{
JUdith G. COHEN*† \\ Berkeley Astronomy Department, University of California, Berkeley \\ Received 1973 April 12; revised 1973 June 18
}

\begin{abstract}
Observations of the equivalent widths and radial velocities of the interstellar lines of Ca II, $\mathrm{Na}$ I, $\mathrm{CH}$, and $\mathrm{CH}^{+}$are presented for 30 stars. A special effort was made to observe stars behind or within dense clouds with up to 3 mag of absorption. The interstellar lines in the cloud stars are compared with those in a group of highly reddened supergiants whose reddening arises from a large distance, i.e., a long path length through relatively thin material. The atomic interstellar lines of the cloud stars are weak compared to those of supergiants with the same color excess; the molecular lines are of comparable strength in the two groups, except that the ratio $n_{\mathrm{CH}} / n_{\mathrm{CH}}+$ is larger in the cloud stars. The ionization equilibrium is discussed, and it is concluded that the deficiency of atomic $\mathrm{Ca}$ and $\mathrm{Na}$ relative to hydrogen in the clouds is about a factor of 100 larger than in the supergiants.
\end{abstract}

Subject headings: abundances, nebular — interstellar matter — molecules, interstellar nebulae

\section{INTRODUCTION}

The interstellar medium is currently a subject of great interest. Since the discovery of complex interstellar molecules, there has been a deluge of observations by radio astronomers concerning their distribution and the physical properties of the clouds within which they are formed. Intense theoretical activity has begun on understanding the formation of these molecules, the thermal balance of the clouds and other related problems. The suggestion by Field, Goldsmith, and Habing (1969) and by Mészáros (1972) that the grains in the clouds may grow at the expense of the heavy elements in the gas provides additional motivation for the present study. We attempt to provide observations of the optical interstellar lines for stars embedded in or behind dark clouds. Adams's (1949) pioneering survey concentrated on distant supergiants, as galactic structure was a major concern of the time. We concentrate on observing nearby stars which are highly reddened and located within dense clouds of absorbing material that can be seen on the Palomar Sky Survey. Tantalizing suggestions of peculiar optical interstellar lines in such clouds exist in the literature (Greenstein and Struve 1939, for example).

In order to avoid a multitude of problems, including a lack of detailed knowledge of the ionization equilibrium, we compare the behavior of the optical interstellar lines in a group of nearby stars with large color excesses located in or behind dark clouds (subsequently called the cloud stars) to the interstellar lines seen in distant, highly reddened supergiants, whose reddening arises from a long path length through an average of the interstellar medium (which we call the thin interstellar medium). The mean density of the thin interstellar medium must be quite small compared to that of the dense clouds, and the latter are sufficiently rare that the line of sight to a supergiant probably does not pass through such a dense cloud. We know that equal

* Guest observer, Hale Observatories, operated by the Carnegie Institute of Washington and the California Institute of Technology.

$\uparrow$ Present address: Kitt Peak National Observatory, Tucson, Arizona. 
color excesses in a cloud star and a supergiant imply roughly equal column densities of dust between the stars and the observer; we will later assume that this also implies equal column densities of gas in front of the two stars.

\section{SELECTION OF STARS}

In order to find stars behind dense clouds, we used the catalog of dark clouds by Lynds (1962), considering only clouds of opacity 5 and 6 (i.e., the clouds with the greatest obscuration) with declinations between $-30^{\circ}$ and $+50^{\circ}$ (corresponding to the limits of the coudé of the 100-inch telescope). The Smithsonian Astrophysical

TABLE 1

PROGRAM STARS

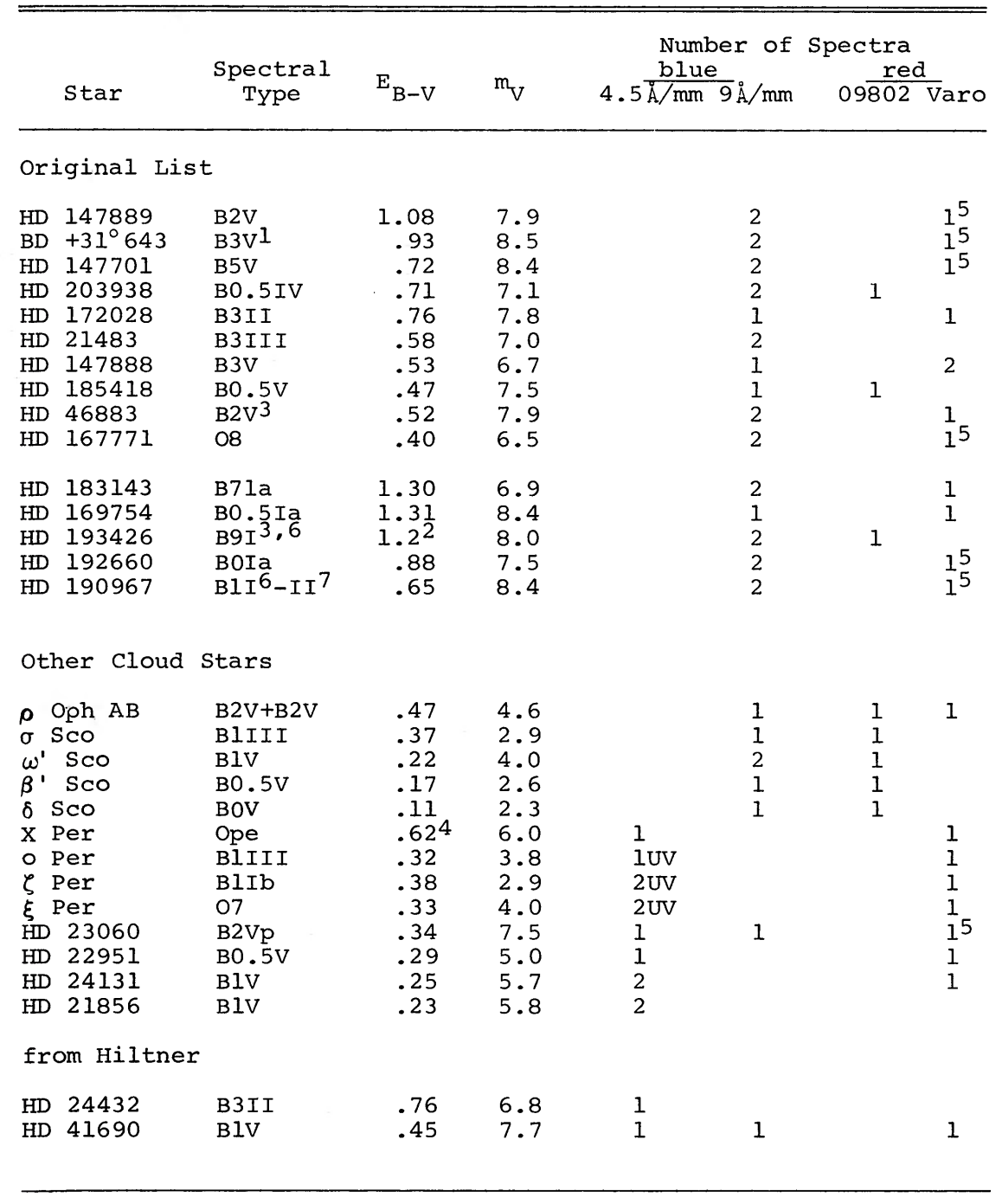

References and Notes:

1. Strom - private communication

2. Crossley scans

3. High dispersion spectra

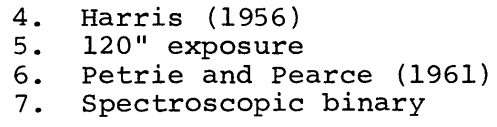

4. Harris (1956)

5. $120 "$ exposure

6. Petrie and Pearce (1961)

7. Spectroscopic binary 
Observatory Star Catalog (1966) was searched for O and B stars brighter than $m_{v}=9.0$ whose positions were close to those of the centers of the clouds. A list of 90 candidates was thus obtained. Many of these stars had $U B V$ colors (Blanco et al. 1970) typical of unreddened stars, which indicated that they were located in front of the cloud, rather than behind it. Those stars without broad-band photometry were observed with the Crossley 36-inch $(91-\mathrm{cm})$ telescope of Lick Observatory, using a dualchannel photoelectric scanner with a $40 \AA$ bandpass.

Seventeen stars remained after eliminating all stars with $E_{B-V}<0.5$ mag. Of those, two subsequently were found to be of spectral type B9, but observations of one are of some interest. Two others, which were of spectral type B8 and quite faint, were never observed. It is gratifying that the most heavily reddened stars in the well-known clouds such as the $\rho$ Oph complex, the II Per association, and the Taurus cloud turned up on the list of candidates. HD 147701, which is in the $\rho$ Oph complex, was added to the original list after it was discovered that the magnitude of 9.4 given in the Smithsonian Astrophysical Observatory Star Catalog (1966) is a misprint, and its visual magnitude is actually 8.4. Additional, less heavily reddened stars in the $\rho$ Oph cloud and the II Per region were observed. Two moderately reddened stars from Hiltner's (1956) survey of O and B stars were also observed as they are bright and conveniently located in the sky. The 30 stars for which observations were made are listed in table 1. The $U B V$ colors and spectral types are from Blanco et al. (1970) unless otherwise noted; color excesses were then derived using the calibration of $B-V$ versus spectral type of Schild, Peterson, and Oke (1971). The stars are listed in order of color excess, except that the supergiants in the original list are ordered separately.

\section{SPECTROSCOPIC OBSERVATIONS}

Many of the program stars listed in table 1 are quite faint, and a compromise must be made between the amount of telescope time available and the resolution desired. Because most of these stars are in clouds with at least 1 mag of absorption, we expected that their interstellar lines would have at most one major component, and perhaps other components weak compared to that resulting from the cloud. We therefore observed these stars at dispersions insufficient to resolve the component structure. The blue spectra (all baked IIaO) had dispersions of 9 and $4.5 \AA \mathrm{mm}^{-1}$ for the brightest stars. All the blue spectra were taken at the coudé of the 100 -inch $(254-\mathrm{cm})$ telescope at Mount Wilson, with the exception of the stars brighter than $m_{v}=5.0$, which were observed with the coudé auxiliary telescope (CAT) feed to the coudé of the 120-inch $(305-\mathrm{cm})$ telescope at Lick Observatory. The red plates fall into several categories. A small number of 09802 emulsion plates (dispersion $18 \AA \mathrm{mm}^{-1}$ ) were taken at the 100-inch telescope. Most of the red plates were taken with the CAT using a Varo image-intensifier system (built by Dr. G. H. Herbig) and 103aD emulsion plates with dispersions of 9 and $16 \AA \mathrm{mm}^{-1}$. A small number of spectra were taken with the Varo image-intensifier system using the 120-inch telescope, through the kindness of Dr. R. Kraft. 09802 spectra for the Scorpius stars were taken at the coudé of the 60 -inch $(155-\mathrm{cm})$ telescope of Cerro Tololo Inter-American Observatory in November of 1972. In addition, for the brightest stars in the II Per cluster, ultraviolet exposures (4.5 $\AA \mathrm{mm}^{-1}$ dispersion) down to $3200 \AA$ were obtained using a UG5 filter at the coudé of the 100 -inch telescope. All these spectra were secured in various observing sessions from February 1972 to February 1973. Also two IN exposures of $\zeta$ Per taken by Dr. L. Houziaux on 1960 November 29, and 1961 January 6, were located in the vaults of the Hale Observatories. We indicate in the final columns of table 1 the available spectroscopic material for each star.

All the blue spectra were measured with an oscilloscope Grant machine for radial velocities of the interstellar features $\left(V_{\text {int }}\right)$. The dispersion is so high that the stellar 
TABLE 2

VELOCITIES AND EQUIVALENT WIDTHS OF INTERSTELIAR LINES

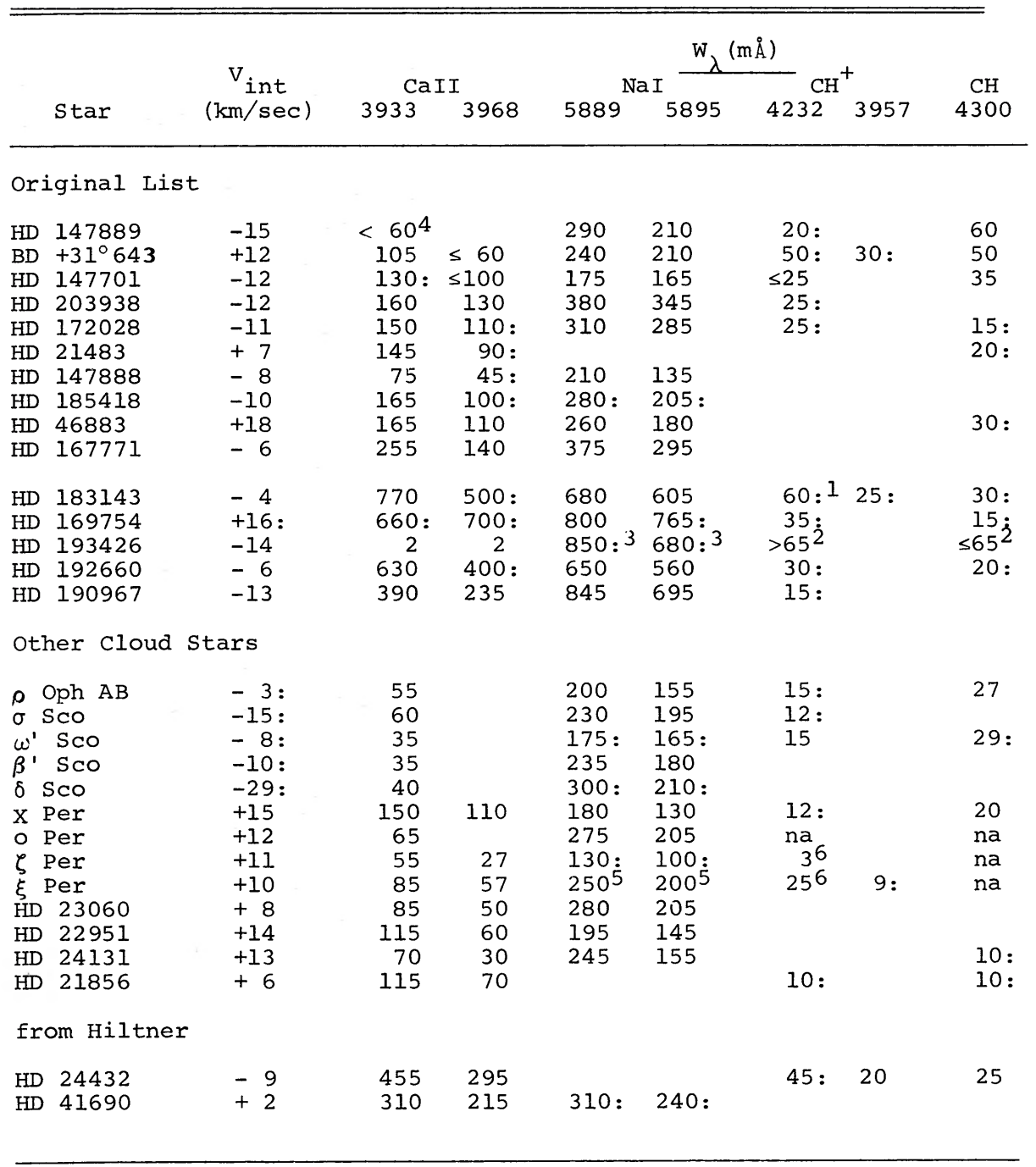

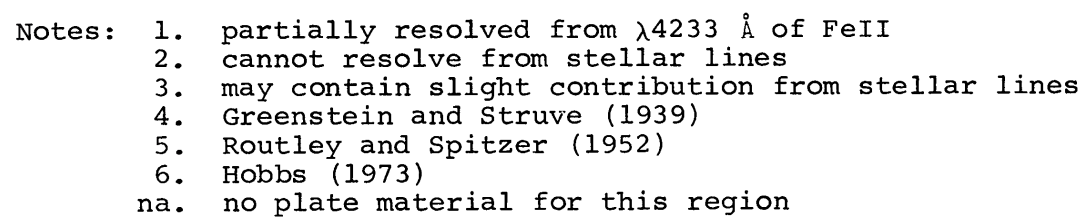

features are too broad to permit an accurate measurement of the stellar radial velocity. We estimate the accuracy of our measurement of $V_{\text {int }}$ from the $\mathrm{H}$ and $\mathrm{K}$ lines of Ca II to be $\pm 3 \mathrm{~km} \mathrm{~s}^{-1}$. In those cases where Adams (1949) has measured $V_{\text {int }}$, the agreement with our measurements is quite good. In all cases where the interstellar molecular lines of $\mathrm{CH}$ and $\mathrm{CH}^{+}$are definitely present, the radial velocity determined from these features is the same as that determined from the atomic interstellar lines to within the above quoted uncertainty. Our measurements of $V_{\text {int }}$, corrected for the Earth's orbital motion, are listed in the second column of table 2. The stellar radial 
velocities, as determined from our own poor measurements or from the Bibliography of Stellar Radial Velocities (Abt and Biggs 1972), are sufficiently different in nearly all cases that there can be no doubt that the lines of $\mathrm{Ca}$ II and $\mathrm{Na} I$ are interstellar.

The spectra were traced using the computer-assisted microphotometer of the Lick Observatory designed by Dr. Lloyd Robinson. Equivalent widths were measured for all interstellar features on the spectra. It is difficult to assess the accuracy of these measurements, as some of the plates are slightly underexposed. Results for cases where more than one plate of a star is available and comparison of our measurements for $\mathrm{H}$ and $\mathrm{K}$ of $\mathrm{Ca}$ II with those of Spitzer, Epstein, and Li Hen (1950) (based on Adams's [1949] spectra) suggest that except for the weaker features $\left(W_{\lambda}<40 \mathrm{~m} \AA\right)$ our values of $W_{\lambda}$ are accurate to within \pm 15 percent. Equivalent widths of the weaker features are probably correct to $\pm 10 \mathrm{~m} \AA$. Errors in equivalent widths of the D lines from the image-tube plates, especially those at $16 \AA \mathrm{mm}^{-1}$, may be slightly larger. The measurements of $W_{\lambda}$ for the interstellar lines $\mathrm{H}$ and $\mathrm{K}$ of $\mathrm{Ca}$ II, $\mathrm{D}_{1}$ and $\mathrm{D}_{2}$ of $\mathrm{Na}$ I, $\lambda 4300$ of $\mathrm{CH}, \lambda \lambda 4232$ and 3958 of $\mathrm{CH}^{+}$are given in table 2 . If no entry is given for the molecular lines, the line was not detected. Upper limits for detection are $15 \mathrm{~m} \AA$ for the $4.5 \AA \mathrm{mm}^{-1}$ spectra and $25 \mathrm{~m} \AA$ for the $9 \AA \mathrm{mm}^{-1}$ spectra. If no entry is given for $\mathrm{H}$, the line is lost in $\mathrm{H} \epsilon$; no entry for the $\mathrm{D}$ lines implies that no spectra were available in that region. In table 3 we give additional data for the doublet of $\mathrm{Na}$ I at $3302 \AA$ and the Ti II line at $3383 \AA$, which were observed in three stars, and for K I $\lambda 7699$. Those measurements which are particularly unreliable (such as cases where the $\mathrm{Ca}$ II line is badly blended with $\mathrm{H} \epsilon$ ) are indicated by a semicolon.

None of the spectra were sufficiently well-exposed at $3875 \AA$ to search for the $\mathrm{CN}$ lines, with the exception of those taken for the ultraviolet $\mathrm{Na}$ doublet. In those three cases, the upper limit to the strength of $\lambda 3875$ of $\mathrm{CN}$ is $10 \mathrm{~m} \AA$. The resonance lines of Ca I $\lambda 4226$ was searched for in all spectra. There were only three possible detections (HD 167771, BD $+31^{\circ} 643$, and X Per) where the line may be present with equivalent width of about $10 \mathrm{~m} \AA$. Dr. F. H. Chaffee communicates upper limits of $1 \mathrm{~m} \AA$ in $\zeta$ Per, o Per, and $\xi$ Per.

The molecular lines were so weak in general that no information on their profiles could be obtained. The weaker lines of $\mathrm{Ca}$ II had full widths at half-maximum (FWHM) of $0.1 \AA$, which is not greater than the resolution of the spectra. The stronger lines had larger FWHMs of up to $0.8 \AA$. In some cases stellar $\mathrm{H}$ and $\mathrm{K}$ lines were present, but they were so broad that they could be separated from the interstellar components. In no case did we notice a second interstellar component of $\mathrm{Ca}$ II. This implies that no component with $\Delta v>1.5 \mathrm{FWHM}\left(=11 \mathrm{~km} \mathrm{~s}^{-1}\right.$ for weak lines) was present with central intensity of more than a third that of the main component.

We plot in figures 1 and 2 the equivalent widths observed for $\lambda 3933$ of $\mathrm{Ca}$ II and $\lambda 5895$ of $\mathrm{Na}$ I as a function of color excess. Separate symbols are used for the supergiants, the cloud stars, and the two stars added from Hiltner's (1956) list. With the

TABLE 3

Equivalent Widths of Additional Interstellar Lines

\begin{tabular}{|c|c|c|c|c|}
\hline \multirow[b]{3}{*}{ Star } & \multicolumn{4}{|c|}{$W_{\lambda}(\mathrm{m} \AA)$} \\
\hline & \multicolumn{2}{|c|}{$\mathrm{Na} \mathrm{I}$} & \multirow{2}{*}{$\underset{3383.8}{\text { Ti II }}$} & \multirow{2}{*}{$\underset{7699.0}{\mathrm{~K} \mathrm{I}}$} \\
\hline & 3302.4 & 3303.0 & & \\
\hline 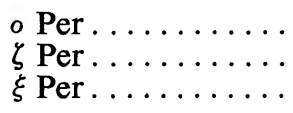 & $\begin{array}{l}50 \\
30 \\
30\end{array}$ & $\begin{array}{l}30 \\
20 \\
15\end{array}$ & $\begin{array}{r}10: \\
6: \\
<10\end{array}$ & $\stackrel{*}{*}+$ \\
\hline
\end{tabular}

* No spectra available for this region. 


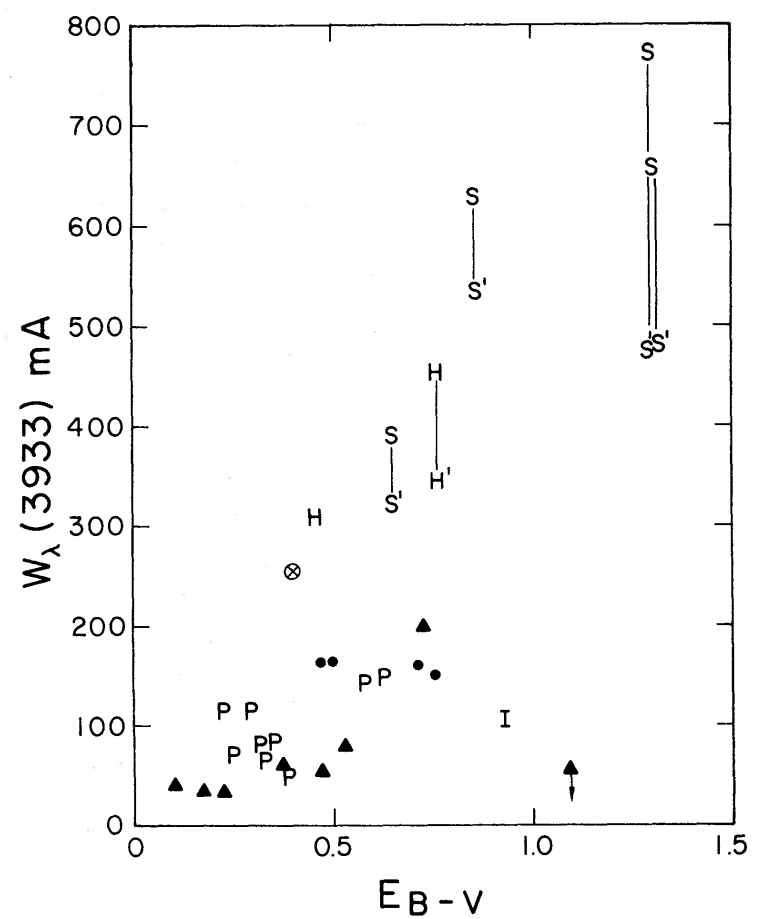

FIG. 1.-The equivalent width of the interstellar $\mathrm{K}$ line of $\mathrm{Ca}$ II as a function of color excess. The symbols are $\mathrm{S}$ (supergiant), $\mathrm{H}$ (additional stars from Hiltner), P (Perseus II association), filled triangles ( $\rho$ Oph cloud), cross (HD 167771), I (BD $+31^{\circ} 643$ in IC 348), and dots (other cloud stars). The primed symbols have been corrected for galactic rotation to $l^{\mathrm{II}}=0$.

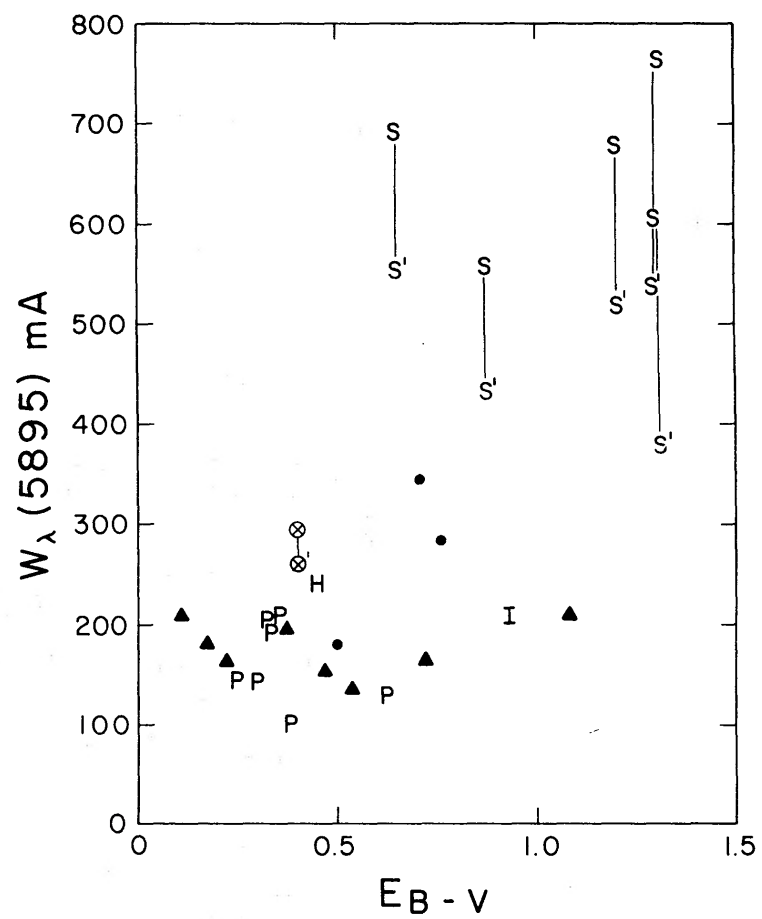

FIG. 2.-Equivalent width of the interstellar line of $\mathrm{Na}$ I at $5895 \AA$ as a function of color excess. Symbols are the same as in fig. 1 . 
exception of the $\mathrm{O}$ star HD 167771, (denoted by $\otimes$ ), the dominant trend is that all of the cloud stars have much weaker interstellar lines at a given color excess than do the supergiants. The relationship is shown more clearly in figure 1 , as the measurements of $\mathrm{K}$ are more accurate than those of $\mathrm{D}_{1}$. Note also that the two stars from Hiltner behave like supergiants rather than cloud stars. This is not surprising, as it is difficult to find stars behind clouds, and therefore a random highly reddened star in the plane is more likely to be distant than to be behind a dense cloud. In the subsequent sections we explore the question of whether this weakness of the atomic interstellar lines in clouds represent a true deficit of heavy elements relative to hydrogen in the clouds, or can be otherwise explained.

\section{COLUMN DENSITIES}

From the data in tables 2 and 3, we derive the doublet ratios (DR) for $\mathrm{K}$ and $\mathrm{H}$ of $\mathrm{Ca}$ II, $\mathrm{D}_{1}$ and $\mathrm{D}_{2}$ of $\mathrm{Na}$ I, and the ultraviolet doublet of $\mathrm{Na}$ I. For doublet ratios greater than 1.1, we use the theoretical curves of growth for a Gaussian velocity distribution within a single cloud (Strömgren 1948) to derive column densities of $\mathrm{Na}$ I and $\mathrm{Ca}$ II. We can derive column densities of $\mathrm{Na}$ I from the standard curves for the $\mathrm{D}$ lines, and from the ultraviolet doublet ratio, by recalling that for weak lines

$$
N=1.13 \times 10^{20} W_{\AA} \lambda_{\AA}^{-2} f^{-1} .
$$

We use the $f$ value for $\lambda 3302$ of Wiese, Smith, and Miles (1969). This gives a second value of the $\mathrm{Na}$ I column density, denoted by $N(\mathrm{Na} \text { I })_{\text {Uv }}$. These two column densities for $\mathrm{Na} \mathrm{I}$ are given in table 4.

We have obtained the same phenomenon as Herbig (1968) did with regard to the ultraviolet $\mathrm{Na}$ I lines; the deduced column densities are much larger than those obtained from the D lines alone. This is either a result of saturation or an incorrect transition probability for the ultraviolet doublet. It would be highly desirable to remeasure the transition probability of the ultraviolet $\mathrm{Na}$ doublet.

In table 5 we list column densities for $\mathrm{Ca}$ II, for $\mathrm{Na}$ I (from the $\mathrm{D}$ lines), and for $\mathrm{CH}$ and $\mathrm{CH}^{+}$. Blank entries denote cases where $\mathrm{DR}<1.1$. The procedure for $\mathrm{CH}$ is identical to that of Herbig (1968). There is no way of directly determining $b$ for the molecular lines from the available observational data, and we adopt the value of $2 \mathrm{~km} \mathrm{~s}^{-1}$, slightly less than $b$ as determined from the atomic lines. The column densities for $\mathrm{CH}^{+}$are calculated from $\lambda 4232$ (the stronger line) only using $f_{\mathrm{el}}=2.2 \times 10^{-2}$ (Solomon and Klemperer 1972), $b=2 \mathrm{~km} \mathrm{~s}^{-1}$, the Franck-Condon factors given by Green, Hornstein, and Bender (1973), and Strömgren's (1948) curve of growth. If we decrease the value of $b$ to $1 \mathrm{~km} \mathrm{~s}^{-1}$, then the molecular column densities for the stronger lines increase by up to a factor of 5 .

We must also worry about the effect of galactic rotation on the interstellar lines seen in the supergiants. (The cloud stars are mostly too close to be affected.) Such effects are undoubtedly present, as the values of $b$ derived are very large. A method of

TABLE 4

Comparison of Na I Column Densities

\begin{tabular}{|c|c|c|}
\hline Star & $\log N(\mathrm{Na} \mathrm{I})_{\mathrm{D}}$ & $\log N(\mathrm{Na} \mathrm{I})_{\mathrm{Uv}}$ \\
\hline $\begin{array}{l}o \text { Per } \ldots \ldots \ldots \ldots \ldots \\
\zeta \text { Per } \ldots \ldots \ldots \ldots \ldots \\
\xi \text { Per } \ldots \ldots \ldots \ldots \ldots\end{array}$ & $\begin{array}{l}12.5 \\
12.2 \\
12.6\end{array}$ & $\begin{array}{l}13.9 \\
13.5^{*} \\
13.5^{*}\end{array}$ \\
\hline
\end{tabular}

$*$ We assume $\mathrm{DR}=2$. 
TABLE 5

COLUMN DENSITIES

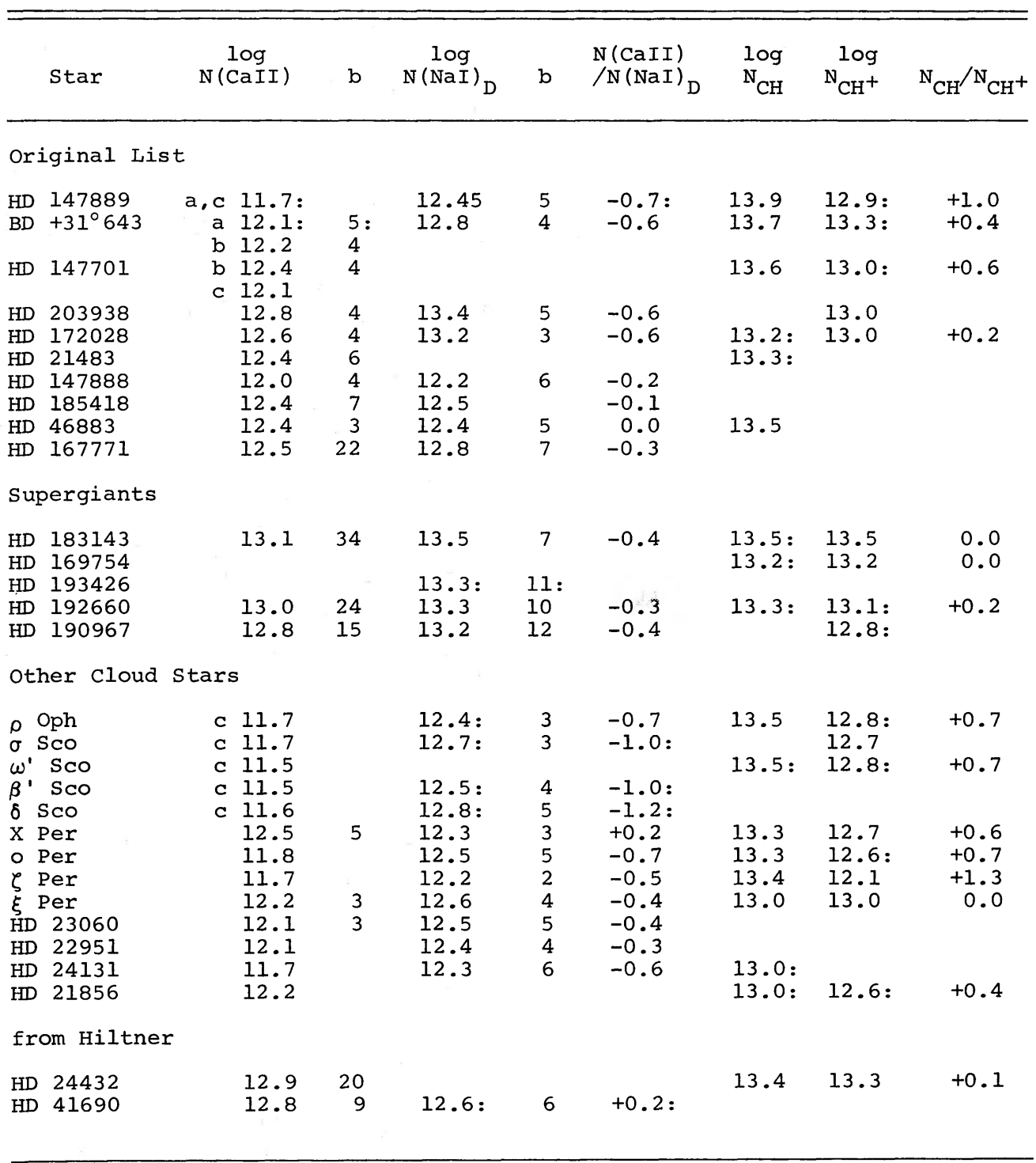

Notes: a. assumes $w_{\lambda}$ is upper limit

b. assumes $\mathrm{b}=4 \mathrm{~km} / \mathrm{sec}$

c. assumes $\mathrm{DR}=2$

correcting the observed $W_{\lambda}$ for galactic rotation is discussed by Spitzer (1948). We have assumed that the external velocity distribution of the clouds has a velocity dispersion $\left(b_{e, 0}\right)$ of $10 \mathrm{~km} \mathrm{~s}^{-1}$. We approximate Spitzer's formula by

$$
C=\frac{W_{\lambda}(l=0)}{W_{\lambda}(l)}=\frac{b_{e, 0}\left[1-\exp \left(-\alpha E_{B-v} / b_{e, 0}\right)\right]}{b_{e}\left[1-\exp \left(-\alpha E_{B-v} / b_{e}\right)\right]}
$$

and

$$
b_{e}=b_{e, 0}+\operatorname{Ar} \frac{\lambda}{c} \sin \left(2 l^{\mathrm{II}}\right),
$$


where $\alpha=1.2 \mathrm{~A}$ per mag for $\mathrm{D}_{1}$ and $\mathrm{D}_{2}$, and $\alpha=0.6 \mathrm{~A}$ per mag for $\mathrm{H}$ and $\mathrm{K}$. Spitzer (1948) and Münch (1968) find that these formulae are in good agreement with the observational data.

Although this correction was derived for $\bar{W}=\frac{1}{2}\left[W_{\lambda}\left(\mathrm{D}_{1}\right)+W_{\lambda}\left(\mathrm{D}_{2}\right)\right]$, we have used it for each component separately. The corrections have been calculated for each of the supergiants, HD 167771, and the two stars from Hiltner. The minimum value of $C$ is 0.63 for HD 183143, which has the maximum range in permitted radial velocity of gas along the line of sight, 0 to $\operatorname{Ar} \sin \left(2 l^{\mathrm{II}}\right) \mathrm{km} \mathrm{s}^{-1}\left(=15 \mathrm{~km} \mathrm{~s}^{-1}\right.$ for this star). The corrected $W_{\lambda}$ are plotted in figures 1 and 2 . Because the distribution of random velocities of clouds appears to be better represented by an exponential velocity distribution than by a Gaussian, we may then derive the column density from the corrected $W_{\lambda}$, the doublet ratio, and the curve of growth for an exponential velocity distribution given by Münch (1968).

Another approach to this problem is to assume that the line of sight intersects sufficiently many clouds randomly distributed in velocity within the range permitted by galactic rotation that we may use the curve of growth for a single cloud with a Gaussian velocity distribution whose velocity dispersion is $\left(b_{e, 0}{ }^{2}+b^{2}{ }_{\text {gal rot }}\right)^{0.5}$ or an exponential velocity distribution with $b_{\text {eff }}=b_{e, 0}+b_{\text {gal rot }}$. Since there are eight clouds $\mathrm{kpc}^{-1}$ (Münch 1968), this will not be a bad approximation for supergiants about $1 \mathrm{kpc}$ away along the local arm.

Each of these three methods of deriving the column densities for the supergiants gives results averaged over this group of stars consistent to \pm 0.1 dex in $\log N$ (Ca II) and \pm 0.3 dex in $\log N(\mathrm{Na} \mathrm{I})$. Of course, the values of the velocity dispersion obtained differ greatly among these methods. We therefore list in table 5 the parameters for the supergiants derived from the single-cloud curve of growth with a Gaussian velocity distribution. A similar correction procedure was tried for the molecular lines. As they have low central optical depths, the saturation is sufficiently small that any correction to the column density is not more than 0.1 dex.

The difference between the supergiant and the cloud atomic interstellar lines for large values of $E_{B-V}$ could be understood purely as a curve-of-growth effect if peculiar conditions prevail. One such case would be if the interstellar lines of the cloud stars are actually a superposition of those of a large number of small clouds whose random velocity dispersion is about $3 \mathrm{~km} \mathrm{~s}^{-1}$ and whose internal turbulent or thermal velocity dispersion is much less than the random motions of the cloud. Another requires a Gaussian velocity distribution which is truncated more than 2 half-widths from the average velocity. However, the conditions required are not those generally thought to prevail in such clouds. Furthermore, the atomic cloud lines (i.e., the part of the line arising in the cloud itself) are even weaker (hence less subject to such corrections) than the numbers in table 2 indicate, as no correction has been made for the material between us and the dense cloud.

Thus the effects of galactic rotation and differing velocity dispersions probably do not eliminate the contrast between the weak atomic lines of the cloud stars and the stronger atomic lines of the supergiants. However, the column densities derived for the supergiants are less accurate because of the assumptions involved in the correction for galactic rotation.

We note that the interstellar molecular lines of stars in clouds continue to be as strong as those in supergiants at large values of $E_{B-V}$ except that the ratio $\mathrm{CH} / \mathrm{CH}^{+}$ changes. An average of the value of the ratio $\log \left(\mathrm{CH} / \mathrm{CH}^{+}\right)$for the cloud stars is +0.6 , while for the supergiants and the stars from Hiltner (1956) it is +0.1 . This systematic difference is larger than any possible error.

The upper limits to the column density of $\mathrm{Ca}$ I as derived from $\lambda 4226$ are $N\left({ }_{\mathrm{CaI}}\right) \leq$ $0.4 \times 10^{10} \mathrm{~cm}^{-2}$ for $\xi$ Per, o Per, and $\zeta$ Per. For the stars with spectra at $4.5 \AA \mathrm{mm}^{-1}$, $N(\mathrm{Ca} \mathrm{I}) \leq 5 \times 10^{10} \mathrm{~cm}^{-2}$. For the other stars, $N(\mathrm{Ca} \mathrm{I}) \leq 10^{11} \mathrm{~cm}^{-2}$. The marginal 
detections listed in $\S$ III correspond to $N(\mathrm{Ca} \mathrm{I})=4 \times 10^{10} \mathrm{~cm}^{-2}$. These numbers do not depend on $b$, the velocity parameter, as the line must be very weak.

\section{IONIZATION EQUILIBRIUM}

We must now consider whether the decrease in strength of atomic interstellar lines between the stars embedded in clouds and the supergiants can be explained by changes in the ionization equilibrium. Also, we must show that the dominant contribution to the interstellar lines seen in the cloud stars arises from the cloud and not from the intervening medium.

We assume that the ionization equilibrium is determined by photoionizations and recombinations only. Then the concentration of an atom $\left(n_{0}\right)$ and its ion $\left(n_{i}\right)$ are given by

$$
n_{i} n_{e} / n_{0}=\Gamma / \alpha
$$

where $\alpha$ is the number of recombinations per $\mathrm{cm}^{3}$ per second of ions and electrons and $\Gamma$ is the probability of photoionization of an atom in the ground state per second. To calculate $\Gamma$ at a given point, we require the radiation density at that point. Consider a star embedded in a cloud. At any point, the radiation density is the sum of that due to the general galactic field, that of the star, and that of the stars in the cloud. Let $d$ be the distance along the line of sight as measured from the star; $R$ is the radius of the cloud; $\rho$ the density of the cloud; $\tau_{c}$ is the optical depth of the cloud in front of the star at $1000 \AA$. We assume that the effective optical depth varies as $\lambda^{-1}$, and that outside of the cloud an optical depth at $1000 \AA$ of $13.4 E_{B-V}$ is produced by interstellar

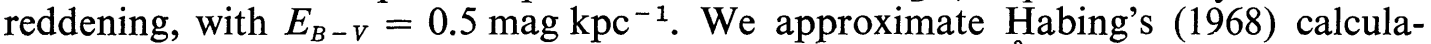
tion of the general galactic field by $U_{g}{ }^{0}=34 \times 10^{-18} \mathrm{ergs} \AA^{-1} \mathrm{~cm}^{-3}$. Then, if $\lambda^{\prime}=$ $1000 / \lambda$, for $\lambda$ in angstroms,

$$
\begin{array}{ll}
U_{g}(d, \lambda)=U_{g}{ }^{0} \exp \left[-\tau_{c} \lambda^{\prime}(R-d) / R\right] & \text { for } d<R ; \\
U_{g}(d, \lambda)=U_{g}{ }^{0} & \text { for } d>R .
\end{array}
$$

For a star of given radius $r$ and effective temperature, we obtain $F_{v}$ from Carbon and Gingerich (1968). Then in the absence of any extinction, $U_{*}{ }^{0}(d, \lambda)=F_{v}(4 \pi / c) \times$ $\left(c / \lambda^{2}\right)\left(r^{2} / 4 d^{2}\right)$. Therefore

$$
\begin{aligned}
& U_{*}(d, \lambda)=U_{*}^{0}(d, \lambda) \exp \left(-\tau_{c} \lambda^{\prime} d / R\right) \quad \text { for } d<R . \\
& U_{*}(d, \lambda)=U_{*}^{0}(d, \lambda) \exp \left[-\left(\tau_{c}+(d-R) \times 0.5 \times 13.4\right) \lambda^{\prime}\right] \\
& \quad \text { for } d>R(d, R \text { in kpc }) .
\end{aligned}
$$

To compute the radiation field from the stars embedded in the cloud, we consider a cloud containing $N_{i}$ stars of the $i$ th spectral class which contribute to the ultraviolet flux (from $\mathrm{O} 5$ to A0), each suffering an average extinction of $\langle\tau\rangle$ magnitude (at $1000 \AA$ ) and with a mean separation of $s$. In the denser clouds studied by radio astronomers, $N_{i}$ is 0 for all $i$, but for the great complexes in Perseus and Ophiuchus, $N_{i}$ may be nonnegligible. For each spectral class we may derive $U_{*}{ }^{0}(i, d, \lambda)$. Then

$$
\begin{array}{ll}
U_{\text {cloud }}(d, \lambda)=\Sigma_{i} N_{i} U_{*}{ }^{0}(i, s, \lambda) e^{-\langle\tau\rangle \lambda^{\prime}} & \text { for } d<R ; \\
U_{\text {cloud }}(d, \lambda)=U_{\text {cloud }}(d<R, \lambda) \frac{R^{2}}{4(d-R)^{2}} e^{-(d-R) \times 0.5 \times 13.4 \lambda^{\prime}} & \text { for } d>R(d, R \text { in kpc }) .
\end{array}
$$

We have written a computer program which calculates the sum $U_{\text {cloud }}+U_{g}+U_{*}$ as 
a function of $d$ and $\lambda$ for any given effective temperature and luminosity class (hence radius) of the star, set of $N_{i}, R, \tau_{c},\langle\tau\rangle, s$, and $\rho$.

We must then integrate to obtain

$$
\Gamma(d)=10^{-8} h^{-1} \int_{0}^{\lambda_{0}} a_{\lambda} \lambda u_{\lambda} d \lambda,(\lambda \text { in } \AA)
$$

where $a_{\lambda}$ is the photoionization cross-section $\left(\mathrm{cm}^{2}\right.$ atom $\left.{ }^{-1}\right)$. This procedure has been done for $\mathrm{Ca}$ I, $\mathrm{Ca}$ II, and $\mathrm{Na}$ I. We use the same photoionization cross-sections as were used by Herbig (1968). We also use the same values of $\alpha$ (the recombination coefficient) corresponding to $T_{e}=100^{\circ} \mathrm{K}$ for an $\mathrm{H}$ I region and $T_{e}=10^{4}{ }^{\circ} \mathrm{K}$ for an $\mathrm{H}$ II region. The integration is carried out from $504 \AA$ to the threshold wavelength for $d$ less than the radius of the Strömgren sphere produced by the star. This radius is calculated from the values given in Allen (1963) and the assumed density of the cloud $\rho$, as the Strömgren radius is proportional to $\rho^{-0.67}$. The integral is taken from the Lyman limit to the threshold wavelength outside of this region.

From the values of $\Gamma / \alpha$ for $\mathrm{Na}$ I, Ca I, and $\mathrm{Ca}$ II, we may calculate $n(\mathrm{Na} \mathrm{I}) / n(\mathrm{Na})$, $n(\mathrm{Ca} \mathrm{I}) / n(\mathrm{Ca}$ II $)$, and $n(\mathrm{Ca}$ II $) / n(\mathrm{Ca}$ III $)$ given the electron density.

The accuracy of the computer program has been checked by reproducing (with $\langle\tau\rangle=N_{i}=\tau_{c}=s=0$ ) the values of $\Gamma$ tabulated by Herbig (1968) for $\zeta$ Oph to within a factor of 2.5 at all values of $d$. This agreement is very satisfactory, considering that we have used a slightly different stellar and galactic radiation field than did Herbig.

We now face the problem of determining the electron density, which depends mostly on the ionization of $\mathrm{C}$ and $\mathrm{H}$. In the small Strömgren sphere around the star, we know that $n_{e}=n_{\mathrm{H}}$. Werner (1970) has predicted that within a large dense cloud the carbon will be predominantly neutral. However, he ignores the possibility of a significant ultraviolet radiation field from the stars embedded in the cloud. Solomon and Klemperer (1972) have calculated the ionization equilibrium problem for $\mathrm{H}, \mathrm{C}$, $\mathrm{Na}$, and $\mathrm{Ca}$. They too neglect a possible cloud radiation field, but include cosmic-ray ionization of $\mathrm{H}$. They find $n_{e} / n_{\mathrm{H}}$ in the range of $10^{-3}$ to $10^{-4}$ for $\tau_{c}$ up to 6 . Since the major cloud with which we are concerned has many embedded stars, we find that the additional radiation field from the cloud compensates for the extinction of the galactic field. The ratio $n_{e} / n_{\mathrm{C}}$ is proportional to $\left[\Gamma /\left(\alpha n_{\mathrm{C}}\right)\right]^{0.5}$ for electrons from photoionization of carbon only; we therefore take as an approximation that $n_{e} / n_{\mathrm{H}}=10^{-3}$ within the clouds, and that outside of the clouds, in the thin interstellar medium, $n_{e} / n_{\mathrm{H}}=10^{-3}$.

We now construct a model for the most heavily reddened stars in the $\rho$ Oph cloud. To obtain an estimate of the density, we assume that the gas-to-dust ratio is constant, independent of the density. Evidence is given by Savage and Jenkins (1972) that this is valid for $E_{B-V}$ up to 0.4 mag. The most reddened star in this cloud (HD 147889) has $E_{B-V}=1.1$, and the size of the cloud $(1.5$ at $200 \mathrm{pc})$ is 5 parsecs. We know that $n_{\mathrm{H}}=1 \mathrm{~cm}^{-3}$ produces about $1 \mathrm{mag} \mathrm{kpc}-1$ of extinction. Therefore we adopt $n_{\mathrm{H}}=$ $10^{3} \mathrm{~cm}^{-3}$ for this cloud. We assume $n_{\mathrm{H}}=1 \mathrm{~cm}^{-3}$ outside of the cloud. We obtain $\tau_{c}$ from the color excess of the most heavily reddened star. The values $N_{i}$ are obtained by considering the stars from HD 147165 to HD 148605 with declination within $1^{\circ}$ of HD 147889 in Garrison's (1967) study of the members of the upper Scorpius association. We adopt $\langle\tau\rangle=7\left(E_{B-V} \simeq 0.5 \mathrm{mag}\right)$ and $s=2.5 \mathrm{pc}$.

In table 6 we present the values of $\Gamma /\left(\alpha n_{e}\right)$ at a representative point in each region and the predicted column density through the region for $\mathrm{Na} \mathrm{I}, \mathrm{Ca}$, and $\mathrm{Ca}$ II. The column densities are calculated assuming that $\mathrm{Ca}$ and $\mathrm{Na}$ have solar abundances. As the star is a B2 V, the Strömgren sphere has a radius of only $0.13 \mathrm{pc}$.

There are several critical points to be seen from table 6 . Because the electron density is high in the cloud, the calcium and sodium atoms tend to be less ionized than in the 
TABLE 6

IONIZATION EQUILIBRIUM FOR HD 147889

\begin{tabular}{|c|c|c|c|c|c|c|c|}
\hline \multirow[b]{2}{*}{$d(\mathrm{pc})$} & \multicolumn{3}{|c|}{$\Gamma / \alpha_{n e}$} & \multicolumn{3}{|c|}{ Column Density } & \multirow{2}{*}{$\begin{array}{l}\text { REGION } \\
\text { (pc) }\end{array}$} \\
\hline & $\mathrm{NaI}$ & $\mathrm{Ca} \mathrm{I}$ & $\mathrm{Ca}$ II & $\mathrm{Na} \mathrm{I}$ & $\mathrm{Ca} \mathrm{I}$ & $\mathrm{Ca} \mathrm{II}$ & \\
\hline \multicolumn{8}{|c|}{ Strömgren Sphere } \\
\hline $0.13 \ldots$ & $5.2(-1)$ & $1.1(+1)$ & $0.70(-2)$ & $1.9(+14)$ & $2.0(+13)$ & $3.6(+14)$ & $0-0.13$ \\
\hline \multicolumn{8}{|c|}{ Cloud } \\
\hline $3 \ldots$ & $0.66(+1)$ & $0.18(+3)$ & $2.7(-2)$ & $2.0(+15)$ & $8.8(+13)$ & $1.6(+16)$ & $0.1-5$ \\
\hline \multicolumn{8}{|c|}{ Outside Cloud } \\
\hline $\begin{array}{l}7 \ldots \\
100\end{array}$ & $\begin{array}{l}1.2(+4) \\
1.5(+3)\end{array}$ & $\begin{array}{l}0.4(+6) \\
3.5(+5)\end{array}$ & $\begin{array}{l}7.5(+1) \\
3.1(+1)\end{array}$ & $3.8(+11)$ & $5.5(+7)$ & $1.9(+13)$ & $5-200$ \\
\hline
\end{tabular}

Note. $A(B)=A \times 10^{B}$.

normal interstellar medium. Within the cloud, almost all of the calcium is in the form of Ca II, and over 10 percent of the sodium is $\mathrm{Na}$ I. But we still have not reduced the level of ionization to the point where more than 1 percent of the calcium is in the form of CaI. If we had not included the radiation field from the stars in the cloud, we would obtain a larger fraction of the $\mathrm{Ca}$ in the form of $\mathrm{Ca} \mathrm{I}$, as did Solomon and Klemperer (1972).

The conclusion that the general level of ionization is lower in the denser clouds implies that a larger fraction of the $\mathrm{Ca}$ and $\mathrm{Na}$ are in the observable forms (Ca II and $\mathrm{Na} \mathrm{I}$ ). This implies that the decrease in column density of $\mathrm{Na}$ I and $\mathrm{Ca}$ II in the clouds as compared with highly reddened supergiants is much larger than numbers in table 5 indicate. For equal total dust column density [hence by assumption, hydrogen (plus $\mathrm{H}_{2}$ ) column density] the cloud has approximately a factor of 10 less $\mathrm{Na}$ I and $\mathrm{Ca}$ II than does the general interstellar medium. From table 6, we see that this may correspond to as much as a factor $10^{-3}$ less total $\mathrm{Ca}$ and $\mathrm{Na}$ in the cloud. In other words, the depletion of calcium and sodium in the densest clouds that can be studied at optical wavelengths is large.

This is also apparent in another way from table 6. The total column density of $\mathrm{Ca}$ II and $\mathrm{Na}$ I, assuming normal solar abundances, from the cloud region is much larger than the maximum permitted by the observations (table 5), even allowing for a factor of 10 error in the values of table 5 due to possible saturation effects. Thus it is possible that a large part of the interstellar atomic lines seen in stars embedded in dark clouds arises from the normal interstellar material between us and the cloud. This would not be the case for clouds closer than $1 \mathrm{kpc}$ if there were no depletion.

The exceptional behavior of HD 167771 (designated by $\otimes$ in figs. 1 and 2) is now understood. The line of sight tc this $O$ star passes through the periphery of an extensive cloud, but the star is so distant $(d=1.7 \mathrm{kpc})$ that most of the reddening $\left(E_{B-V}=\right.$ 0.4 ) is from the intervening medium rather than the cloud. Hence the atomic interstellar lines of this star fit very well onto the relationship displayed by the supergiants.

Another point to note is that because the density is high, the Strömgren spheres (SS) are very small. Unless the radius of the SS is greater than $1 \mathrm{pc}$, the contribution of that region to the total column density of $\mathrm{Na}$ I or $\mathrm{Ca}$ II will be small. To have an SS that large requires an O5 star embedded in a dense cloud, and there are no such stars in our program. (We ignore the possibility that the $\mathrm{O}$ star vaporizes the grains 
TABLE 7

Average Abundances Relative to Hydrogen

\begin{tabular}{|c|c|c|c|c|c|c|c|}
\hline Cloud Stars & $\begin{array}{l}\log \\
\mathrm{Ca} / \mathrm{H}\end{array}$ & $\log _{\mathrm{Na} / \mathrm{H}}$ & $\log _{\mathrm{CH} / \mathrm{H}}$ & $\mathrm{CH}^{+} / \mathrm{H}$ & $\begin{array}{l}\text { No. of } \\
\text { stars }\end{array}$ & $\mathrm{Ca} / \mathrm{Ca} \mathrm{II}$ & $\mathrm{Na} / \mathrm{Na} \mathrm{I}$ \\
\hline $\begin{array}{l}E_{B-v}>0.9 \ldots \ldots \ldots \ldots \\
0.6<E_{B-v}<0.9 \ldots \ldots \ldots \\
E_{B-V}<0.6(\rho \text { Oph }) \ldots \ldots \\
E_{B-V}<0.6 \text { (II Per) } \ldots \ldots \ldots \\
E_{B-V}<0.6 \text { (other) } \ldots \ldots \ldots \\
\text { Supergiants* } \ldots \ldots \ldots \ldots \ldots\end{array}$ & $\begin{array}{l}-9.7 \\
-9.2 \\
-9.2 \\
-9.2 \\
-9.0 \\
-7.1\end{array}$ & $\begin{array}{l}-8.3 \\
-7.7 \\
-6.8 \\
-7.0 \\
-7.2 \\
-5.1\end{array}$ & $\begin{array}{l}-7.9 \\
-8.2 \\
-7.8 \\
-8.1 \\
-7.9 \\
-8.5\end{array}$ & $\begin{array}{l}-8.7 \\
-8.5 \\
-8.5 \\
-8.6 \\
-\dddot{8.5}\end{array}$ & $\begin{array}{l}2 \\
5 \\
6 \\
7 \\
2 \\
8\end{array}$ & $\begin{array}{r}1 \\
1 \\
1 \\
1 \\
1 \\
30\end{array}$ & $\begin{array}{c}7 \\
7 \\
70 \\
70 \\
70 \\
1.5 \times 10^{3}\end{array}$ \\
\hline
\end{tabular}

* Includes two stars from Hiltner and HD 167771.

and hence increases the local density of $\mathrm{Na}$ and $\mathrm{Ca}$; rather we feel it more likely that the gas and grains are pushed away from the star by radiation pressure.)

When considering the expected strength of interstellar $\mathrm{Ca} \mathrm{I}$, we see from table 5 that the SS region may be important. Our upper limits on $\lambda 4226$ (see $\S$ IV) give the following ratios: $\mathrm{Ca}$ II $/ \mathrm{Ca}$ I $>1.6 \times 10^{2}$ (o Per, $\zeta$ Per, $\xi$ Per); $\mathrm{Ca}$ II $/ \mathrm{Ca}$ I $>0.4 \times 10^{2}$ (other cloud stars); $\mathrm{Ca}$ II $/ \mathrm{Ca}$ I $>3 \times 10^{2}$ (supergiants). These limits are satisfied by the entries of table 5. Recall that the three possible detections of $\lambda 4226$ of Ca I (see $\S$ III) were in HD 167771 where the line could be coming from the SS region of this O star (which is itself probably not in a dense cloud), in X Per (again an O star with a large SS region), and in $\mathrm{BD}+31^{\circ} 643$, which is in the central part of a very dark cloud, where the other stars in IC 348 are A stars, rather than of earlier spectral class. Although we are not willing to assert that $\mathrm{Ca}$ I definitely has been detected, at least the possible detections are in accord with our expectations from considerations of ionization equilibrium. If the radiation field from the cloud had not been included, so that $\mathrm{Ca}$ I/Ca II were much larger, then the $\mathrm{Ca}$ I line should have been seen. The fact that it has not been seen is evidence for the importance of the radiation field of the stars embedded in these clouds.

We now compute the average abundances relative to hydrogen, shown in table 7 . Averages are made for the supergiants and for several intervals of color excess of the cloud stars. We obtain the hydrogen column density from the L $\alpha$ data of Savage and Jenkins (1972) and Morton, Jenkins, and Macy (1972). In the majority of cases, where such data are not available, we use the relationship between color excess and $N(\mathrm{H})$ given by Savage and Jenkins. We are thus forced to assume that $\left[N(\mathrm{H})+2 N\left(\mathrm{H}_{2}\right)\right]$ is constant for all of the stars with $E_{B-V}>0.5 \mathrm{mag}$. For some of these regions, $21-\mathrm{cm}$ profiles are available with a beam size of 0.6 from Heiles (1973); however, for a star embedded in a cloud, we do not know how much of the $21-\mathrm{cm}$ profile arises from $\mathrm{H}$ I gas behind the star. Furthermore, the $21-\mathrm{cm}$ line is saturated. The optical depth can be obtained from $21-\mathrm{cm}$ absorption features against extragalactic sources, and it is not greater than 3 (Heiles 1973). This implies that the total column density from $21-\mathrm{cm}$ emission, corrected for saturation, is less than the expected column density of hydrogen derived from Savage and Jenkins's relation by about a factor of 2 . The missing hydrogen may be in the form of $\mathrm{H}_{2}$. Since about 10 percent of the hydrogen is $\mathrm{H}_{2}$ in the outer parts of these clouds, as determined by rocket-ultraviolet measurements of $\mathrm{H}_{2}$ absorption against the less reddened stars in Oph and Per (Smith 1973; Carruthers 1970), we may use the calculations of Hollenbach, Werner, and Salpeter (1971) to predict that $2 \mathrm{H}_{2} /\left(\mathrm{H}+2 \mathrm{H}_{2}\right)$ is unity in the central regions of these clouds. The postulated increase in radius of the grains due to depletion of the heavy elements from the gas may also change the optical depth produced by a given column density of grains. Thus we may expect the slope of the relationship $N_{\mathrm{H}} / E_{B-V}$ to change for large $E_{B-V}$ 
if the grain radius changes significantly. Which of these two effects is the more important is not yet clear.

To obtain the entries in the left part of table 7, the $\mathrm{Ca}$ II and $\mathrm{Na}$ I column densities of table 5 are corrected for ionization equilibrium by the factors (taken from table 6) given in the last two columns of table 7. For $E_{B-V}<0.6 \mathrm{mag}$, we assume $n=10^{2}$ $\mathrm{cm}^{-3}$ rather than $10^{3} \mathrm{~cm}^{-3}$. The logarithms of the corrected column densities are averaged to give the final values shown in table 7 .

The numbers in this table are not to be taken as more accurate than a factor of 10 , at least. We do not know the ionization conditions exactly. Our knowledge of the velocity distributions for the clouds and the supergiants is poor, so that our derivation of column densities from observed $W_{\lambda}$ is not very accurate. We do not know what fraction of the line of sight to the supergiants passes through relatively thin clouds, which will effectively decrease the column density of $\mathrm{Ca}$ and $\mathrm{Na}$ relative to $\mathrm{H}$.

The important conclusions are the large depletion of $\mathrm{Na}$ and $\mathrm{Ca}$ in the clouds, and the deficiency of $\mathrm{Ca}$ in the supergiants. It is impossible to determine which of $\mathrm{Ca}$ and $\mathrm{Na}$ is more depleted in the clouds as compared to the supergiants, due to the inaccuracies and assumptions involved in these entries; however, it appears that $n_{\mathrm{Ca}} / n_{\mathrm{Na}}$ does not change by a factor of more than 50 between the clouds and supergiants. The relative change in the $\mathrm{CH} / \mathrm{CH}^{+}$ratio and the approximately constant ratio $N_{\mathrm{CH}^{+}} / N_{\mathrm{H}}$ are of great importance, as the first supports our calculations that the ionization (i.e., $n_{1} / n_{0}$ is lower in the clouds. Although the data on the supergiants are poor, as all of the plates were at $9 \AA \mathrm{mm}^{-1}$, the reader may peruse table 3 of Adams (1949) for further evidence that the lines of interstellar $\mathrm{CH}^{+}$are much stronger than interstellar $\mathrm{CH}$ in the spectra of supergiants.

It is unfortunate that we could not observe CN. Solomon and Klemperer predict a rapid increase in $\mathrm{CN} / \mathrm{H}$ as $n_{\mathrm{CH}} / n_{\mathrm{H}}$ (or the density) increases. Observations of $\mathrm{CN}$, together with $\mathrm{CH} / \mathrm{CH}^{+}$, would help to determine separately the increase in density in the cloud and the total ultraviolet radiation field in the cloud (including extinction of the galactic field by the cloud and also the presence of a radiation field from the stars embedded in the cloud).

\section{CONCLUSIONS AND DISCUSSION}

The observations reported here are the first indication of depletion where the ionization equilibrium is to some extent observed, through the ratio $\mathrm{CH} / \mathrm{CH}^{+}$. Furthermore, by comparing the behavior of interstellar lines in cloud stars and supergiants, some problems are avoided, although others are added. In such dense clouds, it is impossible to hide $\mathrm{Ca}$ and $\mathrm{Na}$ in the unobservable forms ( $\mathrm{Ca}$ III and $\mathrm{Na}$ II). It is also unlikely that $\mathrm{Ca}$ and $\mathrm{Na}$ are present as gaseous molecules in amounts larger than the atomic form. Therefore, we are forced to conclude that $\mathrm{Ca}$ and $\mathrm{Na}$ are missing from the gas to a greater extent in the clouds than the supergiants; if the ionizationequilibrium calculations of $\S \mathrm{V}$ are at all correct, they are almost completely absent from the gas in the centers of dark clouds ( $\mathrm{Ca}$ always being more depleted than $\mathrm{Na}$ ). This fits in well with Carrasco, Strom, and Strom's (1973) inference from polarization data that the grains are larger in clouds-so much so that most of the heavy elements must be on the grains. If we can eventually determine more accurately the ratios $\mathrm{Ca} / \mathrm{Na}$ in the clouds as compared with the thin interstellar medium, we may derive the ratio $\mathrm{Ca} / \mathrm{Na}$ in the seed grains which presumably grow in the dense clouds. This will yield data useful in understanding the origin of the seed grains and the process of grain growth.

We have observed the molecules $\mathrm{CH}$ and $\mathrm{CH}^{+}$in the gas in approximately the expected proportions calculated by Solomon and Klemperer (1972) and by Watson and Salpeter (1972). The molecular species are not missing from the gas, contrary to 
the behavior of atomic $\mathrm{Ca}$ and $\mathrm{Na}$. The increase in the ratio of $\mathrm{CH} / \mathrm{CH}^{+}$in the clouds is predicted by Solomon and Klemperer.

It is unfortunate that we are forced (from $\S$ III onward) to assume a constant ratio of $\mathrm{H}+2 \mathrm{H}_{2}$ to color excess (i.e., to dust), although Watson and Salpeter (1972) believe that $\mathrm{H}$ and $\mathrm{H}_{2}$ cannot significantly adhere to the grains. Rocket-ultraviolet measurements of the $\mathrm{H}_{2}$ band and of $\mathrm{L} \alpha$ for the central stars of dark clouds will be very useful.

I am very grateful to the Hale Observatories for generous allotments of telescope time and to Drs. G. Grasdalen, C. Heiles, S. Strom, Mr. L. Carrasco, and an anonymous referee for helpful comments. Dr. F. Chaffee has discussed his unpublished observational material. The Miller Institute for Basic Research has provided financial support.

\section{REFERENCES}

Abt, H. A., and Biggs, E. S. 1972, Bibliography of Stellar Radial Velocities (Tucson: Kitt Peak National Observatory).

Adams, W. S. 1949, Ap. J., 109, 354.

Allen, C. W. 1963, Astrophysical Quantities (London: Athlone Press).

Blanco, V., Demers, S., Douglass, G. G., and Fitzgerald, M. P. 1970, Pub. U.S. Naval Obs., Ser. 2, Vol. 21.

Carbon, D. F., and Gingerich, O. 1969, in Theory and Observation of Normal Stellar Atmospheres, ed. Owen Gingerich (Cambridge: MIT Press).

Carrasco, L., Strom, S. E., and Strom, K. M. 1973, Ap. J., 182, 95.

Carruthers, G. R. 1970, Ap. J. (Letters), 161, L81.

Field, G. B., Goldsmith, D. W., and Habing, H. J. 1969, Ap. J. (Letters), 155, L149.

Garrison, R. F. 1967, Ap. J., 147, 1003.

Green, S., Hornstein, S., and Bender, C. F. 1973, Ap. J., 179, 671.

Greenstein, J. L., and Struve, O. 1939, $A p . J ., 90,625$.

Habing, H. J. 1968, B.A.N., 19, 421.

Harris, D. L., III. 1956, Ap. J., 123, 371.

Heiles, C. 1973, private communication.

Herbig, G. H. 1968, Zs.f. Ap. 68, 243.

Hiltner, W. A. 1956, Ap. J. Suppl., 2, 389.

Hobbs, L. M. 1973, Ap. J., 181, 79.

Hollenbach, D. J., Werner, M. W., and Salpeter, E. E. 1971, Ap. J., 163, 151.

Lynds, B. T. 1962, Ap. J. Suppl., 7, 1.

Mészáros, P. 1972, Ap. J., 177, 79.

Morton, D. C., Jenkins, E. B., and Macy, W. W. 1972, Ap. J., 177, 235.

Münch, G. 1968, in Nebulae and Interstellar Matter, ed. B. Middlehurst and L. H. Aller (Chicago University of Chicago Press).

Petrie, R. M., and Pearce, J. A. 1961, Pub. Dom. Ap. Obs. 12, 1.

Routley, P. M., and Spitzer, L., Jr. 1952, Ap. J., 115, 227.

Savage, B. D., and Jenkins, E. B. 1972, Ap. J., 172, 491.

Schild, R., Peterson, D. M., and Oke, J. B. 1971, Ap. J., 166, 95.

Smith, A. M. 1973, Ap. J. (Letters), 179, L11.

Smithsonian Astrophysical Observatory Star Catalog. 1966.

Solomon, P. M., and Klemperer, W. 1972, Ap. J., 178, 389.

Spitzer, L. 1948, Ap. J., 108, 276.

Spitzer, L., Epstein, I., Jr., and Li Hen. 1950, Ann d'Ap., 13, 147.

Strömgren, B. 1948, Ap. J., 108, 242.

Watson, W. D., and Salpeter, E. E. 1972, Ap. J., 175, 659.

Werner, M. W. 1970, Ap. Letters, 6, 81.

Wiese, W. L., Smith M. W., and Miles, B. M. 1969, NBS Standard Ref. Data. Ser., Vol. 22. 